\title{
A Review on Highly-Efficient RFID Tag Anti-collision Algorithm
}

\author{
Hao $\mathrm{Li}^{1}$, Jie Yang ${ }^{2}$ and Junan $\mathrm{Di}^{3 *}$ \\ ${ }^{1}$ Straits Institute, Minjiang University, Fuzhou 350121, P.R. China \\ ${ }^{2}$ School of Information Engineering, Wuhan University of Technology, Wuhan 430070, P.R. China \\ ${ }^{3}$ Department of Electronic Information Engineering, Minjiang University, Fuzhou 350121, P.R. China
}

\begin{abstract}
A mixed algorithm based on tag estimation and tag identification is put forward by analyzing the collision problem and the anti-collision algorithm in RFID and combining the advantages of dynamic frame slotted ALOHA algorithm and dynamic binary search algorithm.
\end{abstract}

Keywords: Radio frequency radiation, anti-collision algorithm, aloha algorithm, binary search.

\section{INTRODUCTION}

The common tag anti-collision algorithms include TDMA-based ALOHA serial algorithm and binary search algorithm in RFID system, which will develop their advantages according to the number of tags. But when the number of tags cannot be estimated, the efficiency of a single algorithm will be low. If they can be applied simultaneously with the combination of the advantages of dynamic frame slotted ALOHA algorithm and dynamic binary search algorithm, the collision problem can be more quickly solved based on the non-fixed number of tags.

\section{OVERVIEW OF RFID ANTI-COLLISION ALGO- RITHM}

RFID (radio frequency identification) is a non-contact automatic identification technology emerging in 1990s. It achieves the purpose to automatically identify the target objects through non-contact two-way communication in radio frequency manner. It has been widely applied in the fields of identity identification, manufactory fabrication and logistics management and so forth, which is also the core technology of the newly-developing Internet of Things.

Anti-collision technology is one of the key technologies for signal identification in RFID system. When there is only one tag within the readable range of a reader, it can be read directly. But the physical truth is that there are commonly multiple tags within the readable range of a reader. Under this circumstance of the shared channel with the same frequency, channel collision will occur when multiple tags send signals into the read channel of a reader simultaneously, thus signals mutually interference interfere and data collision generates, resulting in a communication failure between the reader and the tags. There have been many solutions for the collision problem, and the multiple-access method commonly used as a communication technology is the solution for anti-collision technology at present. There are basically four types: Space Division Multiple Access (SDMA), Frequence Division Multiple Access (FDMA), Code Division Multiple Access (CDMA) and Time Division Multiple Access (TDMA). Based on the hardware technology, the first three solve the collision problem by improving hardware condition, which are less practical because of the low utilization rate and relatively high implementation cost. Software method that can be updated easily is generally adopted, namely TDMA-based ALOHA serial algorithm and the binary anti-collision serial algorithm.

\section{ALOHA SERIAL ALGORITHM}

\subsection{ALOHA Algorithm}

ALOHA algorithm is a time division multiple access method deigned for the transmission of interactive computers and a random access method, whose basic principle is as follows: users can send the data frames at any time when needed, and the senders won't get any response when there is a conflict and the conflicting frame is destroyed. Random back-off will be carried out for a while whenever the tag is identified or not. The backoff time is a random number generated by the tag within a certain time randomly. The purpose of avoiding the collision is achieved due to the different random number.

Suppose $\mathrm{s}$ is the throughput rate, to is the time to transmitting a data packet, $g$ is the exchanging data volume, pe is the probability for successful completion, and $\mathrm{x}$ is the frame number sent per second,

The probability of sending $\mathrm{n}$ data frames in $\mathrm{t}$ seconds is: $\mathrm{p}(\mathrm{n})=(\mathrm{xt}) \mathrm{ne}-\mathrm{xt} / \mathrm{n}$ !

The probability of sending information in t0 seconds is: $\mathrm{pe}=\mathrm{e}-\mathrm{g}$ 
So the probability of not sending information in $2 \mathrm{t} 0 \mathrm{sec}-$ onds is: $\mathrm{pe}=\mathrm{e}-2 \mathrm{~g}$

The throughput calculated is: $\mathrm{s}=\mathrm{ge}-2 \mathrm{~g}$

When $g=0.5$, the throughput rate reaches its maximum value, $18.4 \%$.]

ALOHA algorithm has the biggest advantages of simple algorithm principle, relatively low implementation cost and high efficiency when less tags. And its disadvantages are as follows: 1) Applicable to occasions of low instantaneity, only used for read-only tag. 2) The bigger the number of tags, the larger the collision probability.

\subsection{Slotted ALOHA Algorithm and Dynamic Framed Slotted ALOHA Algorithm}

Slotted ALOHA algorithm adopts TDMA and it starts only when the data transmission is always in a synchronous slot to avoid the local collision of ALOHA algorithm, and the time of a collision is shortened to $\mathrm{t}=\mathrm{t}$.

So the throughput of the algorithm is: $\mathrm{s}=\mathrm{ge}-\mathrm{g}$

When $\mathrm{g}=1$, the throughput rate reaches its maximum value, $36.8 \%$.

But when the number of tags keeps increasing at $\mathrm{g}=1$, as all the times of duration in the slot are associated with the possible tags, moreover, there may be only one tag in the readable range of the reader, it might not be read. If the number of tags is too small, it will waste the channel. The nonfixed time slot can be used to solve the problem.

The principle of dynamic framed slotted ALOHA algorithm is to send two time slots of different sizes through the reader, if the number of tags is more, send a request command to increase the number of time slots till a tag is discovered, so as to find and adjust the size of the slot.

\subsection{Frame Slotted ALOHA Algorithm, Dynamic Frame Slotted ALOHA Algorithm and Enhancing Dynamic Frame Slotted ALOHA Algorithm}

Frame slotted ALOHA algorithm takes n time slots as a communication unit, frame. The tag sends information randomly in each frame time slot, so the transmission amount increases, appropriate for the case with larger transmission amount.

But because various frames have the same and fixed length of time slots, if they have a similar slotted ALOHA algorithm, the fixed time slot will directly affect the identification efficiency. So the sending time slot should be dynamically adjusted.

Dynamic frame slotted ALOHA algorithm can adjust the frame length according to idle condition and collision situation in each frame and modify the frame size in each cycle by using a tag estimation function. Tag estimation function is used to calculate the timeslot number required by the next identification circle based on the empty timeslot number, successful timeslot number and collision timeslot number that the reader feedbacks.

The increasing dynamic frame slotted ALOHA algorithm is improved based on the restrictions or identification of number of tags. For example, the grouped dynamic frame slotted ALOHA algorithm limits the response tag number in each frame by grouping. The suggestion of improving the grouping function and many improved methods have been proposed, such as the algorithm adding fractional number and tag grouping number, selection method for $q$ value in Gen2 protocol, but they have the disadvantages of complex algorithm and difficult implementation. So dynamic frame slotted ALOHA algorithm has been more genetically applied at present.

\section{BINARY ANTI-COLLISION ALGORITHM}

\subsection{Binary Tree Search Algorithm}

Binary anti-collision algorithm is regularly composed of rules for the interaction (command and response) stipulated between a reader and multiple tags.

The algorithm implementation is as follows:

1) Request (EPC): request serial number. The reader sends a serial number as a parameter to the tag, the tag compares the parameter with its own serial number which will be returned to the reader if it is less than or equal to the serial number.

2) Select (epc): select the tag. The reader sends a serial number to the tag, and the tag of this serial number will take it as the starting switch.

3) Read-Data: read and write the data. The selected tag sends data to the reader.

4) Unselect: deselect. The tag enters into no answer state.

Binary tree search algorithm was used when comparing the parameter with the standard serial number. Its thought is to keep narrowing the range of the corresponding tag till the only tag is identified through multiple comparisons, and then repeat the above operation cycle to continue to identify the next tag till all the tags are identified. Adaptive binary tree search algorithm, adaptive query tree algorithm and recoverable search algorithm have been improved in search strategy.

\subsection{Dynamic Binary Tree Search Algorithm and the Im- proved Binary Tree Search Algorithm}

As the serial number in the tag also contains additional information sent by the reader, a dynamic binary tree search algorithm is proposed, in order to avoid the transmission of spare parts in the serial number to improve the transmission speed. The serial number in the tag removes the additional information when the reader and the tag interact on each other.

The improved method of binary tree search algorithm includes regressive index algorithm, jumping dynamic search, binary tree algorithm, binary query tree algorithm, binary slotted tree RFID tag anti-collision algorithm, and binary slotted tree algorithm etc., which have improved the identification efficiency to some extent.

5. The Mixed Algorithm Based on ALOHA Serial Algorithm and Binary Search Algorithm 
Due to the simple algorithm and low implementation cost, ALOHA serial algorithms have been commonly used, especially the dynamic frame slotted ALOHA algorithm. When the number of tags is large and bigger transmission data, dynamic frame Aloha algorithm can play its advantage of strong real-time performance. However, ALOHA serial algorithms identify the tags randomly, so the possibility of collision is uncertain, when the collided tags are too many, it will directly lead to the decrease of identification efficiency of the algorithm.

Because the binary search algorithm is tree-based, the selection of tags is predictable, so it has stronger identification capability of tags when there is less number of tags. But because of the need to the request and interactive response of each tag, too many numbers of tags will greatly reduce the identification efficiency of the algorithm.

So, the mixed algorithm of dynamic frame slotted ALOHA algorithm and dynamic binary search algorithm can be adopted when the number of tags is uncertain during the actual processing. The anti-collision problem is divided into tag estimation and tag identification. The relatively simple dynamic frame slotted ALOHA algorithm suitable for occasion of larger numbers of tags is first adopted to estimate the number of tags in the tag estimation as its number of tags may be relatively large. After the estimation, the tags entering into the identification module is less, and then dynamic frame slotted ALOHA algorithm is used for primary tag identification, when the collision occurs, the number of collided tags will be less, and then the binary search algorithm is adopted for the two-step identification. Continue to the next tag estimation and identification, and repeat it till all the tags are completely identified. This mixed algorithm can greatly improve the identification efficiency.

\section{CONCLUSION}

According to the above analysis, ALOHA serial algorithm and binary search algorithm have their own advantages and disadvantages in solving the collision problems, and the mixed algorithm based on ALOHA serial algorithm and binary search algorithm can be applied in solving practical problems, and taking advantages of both can quickly solve the collision problem.

\section{CONFLICT OF INTEREST}

The author confirms that this article content has no conflict of interest.

\section{ACKNOWLEDGEMENTS:}

This paper is the subsidy project of national science foundation key project (project authorization number 50830203, and supported by the State Key Program of National Natural Science of China (Grant No. 51479159); The Education Department of Fujian province science and technology research project (JB12155); Fujian branch of research project of National Occupation Education Research Institute (GZM13012); Project supported by special fund of central government financial support for the development of local colleges and universities (Min Cai-Jiao-Zhi-2014-50).

\section{REFERENCES}

[1] Y. L. Huan, The Internet of Things: detailed annotation of Core Technologies of IFID, Version 2. Beijing: Posts \& Telecom Press, 2012.

[2] X. Wang, "A Study on Binary Tree-based RFID Anti-collision Algorithm", Journal of Communication, no. 6, pp. 49-57, 2013.

[3] W. S. Sun and C. M. Jin, "New-type RFID Dynamic Frame Slotted Aloha Anti-collision Algorithm", Information and Control, no. 4, pp. 233-238, 2014.

[4] Z. L. Liu, "Evaluation on developing level of unban agglomeration derived from resources exploration", Journal of Applied Sciences, vol. 13, no. 21, pp. 4702-4707, 2013.

[5] NTT NTTCOMWARE Corporation. RFID's Present Situation and Development Trend. Beijing: Posts \& Telecom Press, 2012.

[6] D. Bhattacharya and S. Roychowdhury, "A Constrained Cost Minimizing Redundancy Allocation Problem in Coherent Systems with Non-overlapping Subsystems", Advances in Industrial Engineering and Management, vol. 3 no. 3, pp. 1-6, 2014.

[7] D. Shi and H. Sun, Taxonomy and survey of RFID anti-collision protocols. Computer Communications, 2014.

[8] B. Liang, A. Q. Hu, and Y. Zhong, "Novel design for RFID anticollision technique", Journal of Electronicsand Information Technology, vol. 29, no. 9, pp. 25-27, 2014

[9] W. C. Ju and C. F. Yu, An RFID Anti-collision Algorithm Based on Dynamic Binary System. Fudan University, 2014.

Received: September 16, 2014

Revised: December 23, 2014

Accepted: December 31, 2014

(C) Di et al.; Licensee Bentham Open.

This is an open access article licensed under the terms of the (https://creativecommons.org/licenses/by/4.0/legalcode), which permits unrestricted, non-commercial use, distribution and reproduction in any medium, provided the work is properly cited. 